\title{
Métodos de casos para el aprendizaje de la Entomología Forense
}

\author{
Jose Antonio Núñez Rodríguez ${ }^{1}$
}

\section{RESUMEN}

El estudio tuvo como objetivo presentar una propuesta didáctica basada en los métodos de casos para el aprendizaje de la entomología forense. Esta es una disciplina que tiene como principal objetivo establecer una data de muerte, de allí surge la importancia del módulo de Tanatología y Antropología Forense del Diplomado en Ciencias Forenses y Criminalística de la Universidad José Antonio Páez, el cual persigue que los participantes puedan determinar la data de muerte mediante el estudio de la entomofauna de interés forense. La metodología se enmarcó en un diseño no experimental transeccional descriptivo. La población estuvo conformada por 24 participantes del Diplomado, Cohorte IX-Diciembre 2015. Se diseñó un caso, donde los estudiantes debían determinar la data de muerte y fundamentar sus conclusiones en pequeños grupos. A través de la técnica de la observación se evidenció que los grupos lograron resultados similares a la data de muerte determinada por el docente durante el desarrollo del caso, estableciendo el Intervalo Postmortem Mínimo en un lapso de seis días, concluyendo que la primera mosca llego al cuerpo el día 06/12/15 entre la una y las dos de la tarde. Se concluye que los estudiantes desarrollaron las habilidades analíticas al identificar el problema, la toma de decisiones al proponer una solución, habilidades para la comunicación oral y escrita, defendiendo y argumentando sus conclusiones, reconociendo sus errores y debilidades; apoyados en el aprendizaje colaborativo.

Palabras claves: Data de muerte, estrategia de aprendizaje, insectos.

Recibido: 21 de febrero de 2016

Aceptado: 19 de octubre de 2016

1 Departamento de Ciencias Morfológicas y Forenses. Escuela de Ciencias Biomédicas y Tecnológicas. Facultad de Ciencias de la Salud, Universidad de Carabobo. Valencia. Venezuela. Correo electrónico: joannuro@gmail.com 


\title{
Case method for the learning of Forensic Entomology
}

\begin{abstract}
This study aimed to present a didactic proposal based on the case method for the learning of forensic entomology. This discipline intends to establish a death data, thus, the Tanatology and Forensic Anthropology of the Diploma course in Forensic and Forensic Sciences of the Universidad José Antonio Páez becomes highly important. The course allows students to determine a death data through the study of the entomofauna of forensic interest. The methodology was framed in a nonexperimental, descriptive transactional design. The population was made up by 24 participants from the Diploma course, cohort IX-December 2015. A case where the students had to determine the death data, and to justify their conclusions in small groups was designed. Through observations it was evident that the groups achieved the expected results, establishing the minimum Postmortem interval in a lapse of six days, concluding that the fly approached the body on 12/06/2015 between 1 and $2 \mathrm{pm}$. It was concluded also that the analytical skills to identify the problem, the decision making in proposing solutions, both written and oral skills, defending and arguing their conclusions, acknowledging their errors and weaknesses; supported by a collaborative learning.
\end{abstract}

Key words: Death data, learning strategy, insects. 


\section{INTRODUCCIÓN}

La entomología forense (EF) se ha definido como una disciplina auxiliar de las ciencias forenses, mediante el uso de insectos como evidencia; sin embargo, el término puede incluir otros artrópodos como escorpiones, arañas y cangrejos (Gennard, 2007). Por ejemplo, Mavárez y otros, (2005) resaltan la importancia de la EF en aquellos casos donde el cuerpo se encuentra en un estado avanzado de descomposición, justo en los fenómenos destructores del cadáver los métodos de la patología clásica para datar el tiempo de muerte son insuficientes, siendo necesario el apoyo de los antropólogos, odontólogos y entomólogos forenses. ¿Cuál es el rol y la función de un entomólogo forense?, es apoyar al sistema de justicia mediante el análisis y estudio científico de las evidencias entomológicas, cuyos objetivos se resumen en la tabla I.

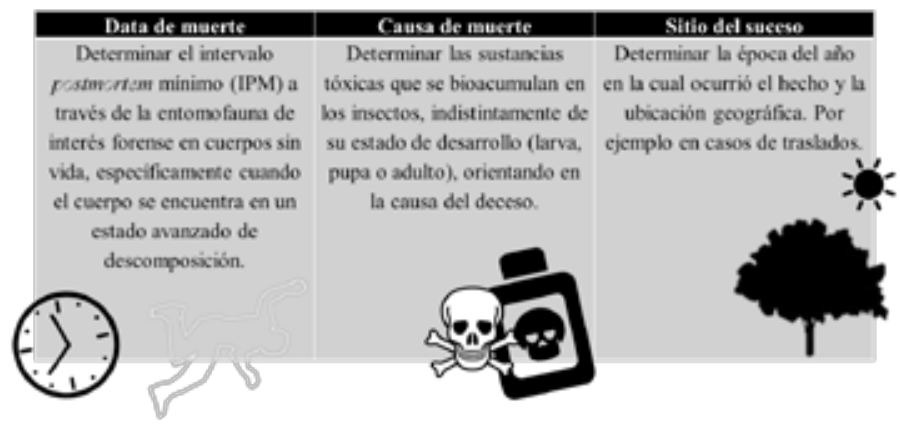

Fuente: elaboración propia

Como el primer objetivo de la EF, es determinar la data de muerte o Intervalo Postmortem Mínimo (IPM), a continuación se define ¿Qué es el IPM?. Se define como los límites de tiempo en los cuales debió ocurrir la muerte de un individuo, siendo el tiempo transcurrido entre el momento último en el que se vio con vida el individuo y el momento en el cual es hallado el cuerpo. Este intervalo proporciona un rango muy aproximado a la realidad, ameritando una colecta de evidencias y resguardo de la cadena de custodia con el fin de obtener resultados óptimos (Sanabria, 2008). Al respecto, la presencia de la entomofauna sobre el cuerpo humano sin vida, permite al entomólogo forense apoyar al médico anatomopatólogo, estableciendo los márgenes mínimos de tiempo de muerte transcurridos desde la llegada de los insectos al cuerpo hasta el momento del hallazgo (Nuñez, 2012).

Pero ¿Cómo un entomólogo lograría datar la muerte de una persona?, como el objetivo principal de la EF es estimar el IPM, se consideran tres métodos: 1) el uso de curvas de crecimiento, 2) matriz de ocurrencia de las especies presentes en el cuerpo humano sin vida y 3) recientemente la tendencia a nivel internacional es usar un tercer método llamado "Horas grados acumulados" (HGA); al respecto Amendt y otros, (2010) establecieron que este método considera en primer lugar identificar las especies presentes en el cuerpo, en segundo los datos de temperatura en el sitio del suceso, incluso datos de cinco a siete días después del hallazgo en el sitio del suceso y tercero obtener los datos de las temperaturas durante los días anterior al hallazgo del cuerpo, mediante una estación meteorológica más cercana al sitio del suceso. La acumulación total de grados horas o días, reflejan el tiempo que ha tardado el insecto en llegar a la fase de desarrollo en que se ha recogido en el lugar del hallazgo.

Antes de aplicar el método "Horas grados acumulados", es necesario conocer ¿Qué es una mosca (díptero)?, su biología e identificación taxonómica; bases conceptuales necesarias para comprender la importancia del EF y su aporte al sistema de justicia. Una mosca es un insecto; es decir, que posee un cuerpo dividido en cabeza, tórax y abdomen, con un par de alas y un par de balancines en el tórax y tres pares de patas. Su pieza bucal es chupadora en el adulto, alimentándose de los fluidos del cadáver. Su ciclo de vida se caracteriza por sufrir una metamorfosis durante su desarrollo, pasando por diferentes estados (formas clásicas de los insectos durante la metamorfosis) y estadios (intervalo de tiempo entre dos mudas sucesivas en un insecto inmaduro) (Ramírez, 2012). El ciclo de vida comprende: huevo, tres instares larvales, prepupa, pupa y adulto (fig. 1). 


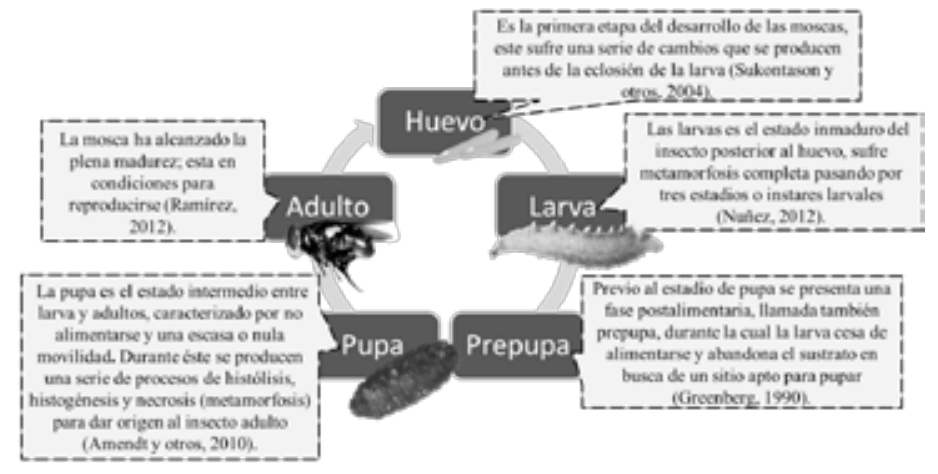

Fig. 1: Ciclo de vida de una mosca y definiciones. Elaboración propia.

En un cadáver podemos encontrar cualquiera de estos estados de desarrollo de una mosca; sin embargo, la EF se apoya principalmente de las larvas para determinar el IPM (fig. 2-A), esto se debe a que las larvas se alimentan de los tejidos del cuerpo humano sin vida, los cuales son desgarrados por las piezas bucales (esqueleto cefalofaríngeo, fig. 2-B). Las larvas presentan un par de espiráculos anteriores y un par posterior (fig. 2-C), los cuales son simples orificios que se conectan con una red traqueal interna por medio de los cuales se lleva a cabo la respiración, es una característica que permite determinar el estadio de desarrollo larval mediante el análisis de las hendiduras espiraculares que se observan en los espiráculos posteriores, pueden variar de una a tres hendiduras. La importancia de esto es que permite indiciar según el número de hendiduras presentes el estadio en el cual se encuentra la larva (tiempo de vida), transformándose en el tiempo que puede llevar una persona fallecida (Nuñez, 2012). Por ejemplo, una hendidura, indica que la larva se encuentra en un primer estadio larvario (Instar I), dos hendiduras (Instar II) y tres hendiduras (Instar III) o larva madura.

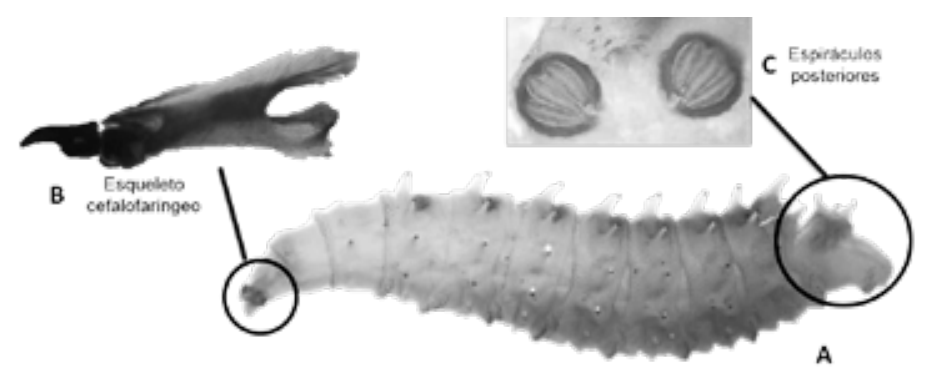

Fig. 2: Larva de Chrysomya albiceps (Diptera: Calliphoridae). A) Larva, B) pieza bucal (esqueleto cefalofaríngeo) y C) vista posterior de la larva, detalle de los espiráculos posteriores. Elaboración propia

El estudio entomológico para la identificación taxonómica de insectos de importancia forense se logra mediante el uso de claves taxonómicas, morfometría geométrica y técnicas moleculares, las cuales consisten en la determinación correcta de la identidad de la especie de insectos, a partir de las características morfológicas, anatómicas y genéticas de los mismos para relacionar al organismo con una categoría taxonómica (Camacho, 2005; Nuñez, 2012). Por ejemplo, en un sitio del suceso se colectan 30 larvas, las cuales mediante una clave taxonómica se separan según su morfología, identificando a las especies Chrysomya albiceps y Chrysomya megacephala; sin embargo ¿Cuál mosca llego primero?, ¿Cómo lograr que el estudiante comprenda la importancia que arroja la determinación del IPM, en un caso forense?.

Al respecto, autores como García, (2005); Bonfigli y otros, (2010); Sebastiany y otros, (2013a); Sebastiany y otros, (2013b); Nuñez, (2015); proponen los métodos de casos como estrategias de enseñanza y aprendizaje en el campo de la criminalística y ciencias forenses, el objetivo es que los estudiantes deben jugar un papel activo, que les permita aplicar sus conocimientos, cuestionar y proponer soluciones a los diferentes casos mediante los métodos aplicados para lograr descubrir, recabar y conformar los medios de pruebas, que les servirán posteriormente al Fiscal del Ministerio Público como fundamento de la acusación; siendo el Juez quien decide la culpabilidad o no del acusado mediante la variedad de medios aportados al proceso, de allí la importancia de la criminalística y ciencias forenses al contribuir al esclarecimiento de la verdad en la investigación del delito.

\section{¿Qué es un método de casos?}

Un caso es la descripción de una situación real, incluso puede ser diseñada, que comúnmente involucra una decisión, un reto, una oportunidad, un problema 
o una cuestión central que afronta una persona (o personas) en su futuro laboral (Garcia y otros, 2012). Los métodos de casos (situaciones problemáticas) en el contexto educativo son estrategias didácticas y de investigaciones basadas en un aprendizaje constructivista, para lograr que el estudiante adquiera y conecte eficazmente el conocimiento teórico y práctico. Al respecto González (2015) señala que los métodos de casos como estrategia, el docente diseña y adapta el caso partiendo tanto del contexto formativo de los estudiantes y sus características, como de los contenidos de aprendizaje que respondan al objetivo buscado.

Este método permite a los estudiantes construir su aprendizaje a partir del análisis y discusión de cada caso, basados en su aprendizaje previo adquirido tanto en su formación académica como en su vida personal; al plantear un caso donde el resultado esperado sea una o varias soluciones, los estudiantes se centran en la toma de decisiones que requiere la solución del problema planteado, fomenta el aprendizaje colaborativo y trabajo en equipo, desarrollando la capacidad de análisis e investigación, el pensamiento crítico, creativo e innovador. En la figura 3, se resumen los aspectos generales de los métodos de casos.

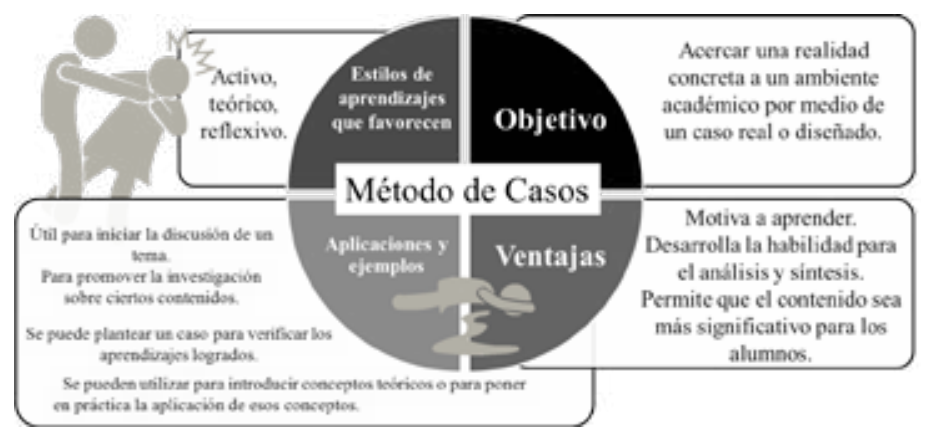

Fig. 3: Resumen del método de casos. Tomado y modificado de Garcia y otros, 2012.

La criminalística y ciencias forenses, dentro de la cual se incluye la EF requieren que los estudiantes logren analizar, discutir e intercambiar argumentos que les garantices la construcción de conocimientos y alternativas para la solución de problemas de interés legal, los cuales se transforman en un entrenamiento para afrontar la realidad laboral. Para Niño (2012), este tipo de estrategia de aprendizaje promueve entre los integrantes del grupo procesos de autoevaluación, favoreciendo la revisión del desempeño estudiantil y el reconocimiento de dificultades; es decir, un método de caso no ofrece las soluciones al estudiante, sino que le entrena para generarlas. Lo que se pretende es entrenar a los estudiantes para encontrar soluciones razonables, la idea es traer la realidad al aula de clases y aportar un método que los haga pensar.

Para lograr comprender los métodos para determinar el IPM, el presente estudio tiene como objetivo presentar una propuesta didáctica basada en los métodos de casos para el aprendizaje de la entomología forense. Es importante destacar, que el entomólogo forense al determinar el IPM, logra aportar una información valiosa para establecer una data de muerte, de allí surge la importancia del módulo de Tanatología y Antropología Forense del Diplomado en Ciencias Forenses y Criminalística de la Universidad José Antonio Páez, el cual persigue dentro de los objetivos que los participantes puedan determinar la data de muerte mediante el estudio de la entomofauna de interés forense.

\section{MATERIALES Y MÉTODOS}

La presente investigación se enmarcó en un diseño no experimental transeccional descriptivo. Es descriptiva porque la misma se realiza a través del contacto con el fenómeno en el estudio y trata de un problema práctico, el cual fue extraído directamente de la realidad (Tamayo, 2003). Donde el plan trazado para la verificación de la problemática planteada respondió a situaciones de análisis sin manipulación alguna de la variable, cuyo comportamiento fue observado y medido en un tiempo único, destacando de tal manera sus rasgos o características más relevantes.

Con el fin de dar respuesta al objetivo propuesto, la estrategia que reúne las características sobre el estudio, 
se apoya en una investigación de campo, que según Bersanelli y Gargantini (2006) es aquella que es aplicada para comprender y resolver alguna situación, necesidad o problema en un contexto determinado, en un lugar y tiempo en que ocurren los fenómenos objeto de estudio. La población estuvo conformada por 24 participantes del Diplomado en Ciencias Forenses y Criminalística de la Universidad José Antonio Páez, Cohorte IX-Diciembre 2015. Durante el estudio se prescindió de la técnica de muestreo, dado que las características de la población reflejan que es medible, cuantificable y accesible en términos de sus integrantes.

Partiendo del objetivo formulado, el tipo y diseño de investigación asumido se adoptó la técnica de la observación directa como instrumento de recolección de información, consistiendo en el registro sistemático, valido y confiable de comportamientos o conductas; es decir la conducta se describe en el momento exacto en que está ocurriendo. Según Rivas (2006), la investigación directa es aquella en que el investigador observa directamente los casos o individuos en los cuales se produce el fenómeno, entrando en contacto con ellos. Aunado del reporte escrito de las conclusiones redactadas y fundamentadas por los participantes.

\section{Diseño y aplicación del caso:}

El caso diseñado lleva por título Determinemos la data de muerte mediante el uso de la entomofauna de interés médico legal y su tema principal es el planteamiento de un presunto homicidio, donde se requiere la aplicación de los conocimientos que aporta la criminalística y ciencias forenses para resolverlo y lograr determinada la data de muerte. El caso fue diseñado mediante una revisión bibliográfica y experiencias personales de diversos investigadores en el área de la entomología forense. El contexto y cuerpo del caso se describió detalladamente, pero de forma precisa la situación problema, organizada en subtítulos para enmarcar cada etapa del proceso y la información que aporta la misma. Se incluyó información de apoyo para ampliar la información proporcionada en el cuerpo del caso como fue una tabla donde se plasman los datos de la temperatura para los días $01 / 12 / 15$ al 14/12/15. Al final del mismo se incluyó la interrogante del caso al plantear que determinen el IPM y redacten sus conclusiones (fig. 4).

Antes de aplicar el caso se dictó el componente teórico - práctico (fundamentos sobre EF, protocolos para la colecta y análisis de insectos, ejercicios para calcular el IPM, fenómenos cadavéricos, tanatología y antropología forense) necesario para el entendimiento de la entomología aplicada al campo legal. Se consideró los conocimientos previos de los estudiantes para el diseño del caso. El caso se presentó a los estudiantes mediante un texto con una duración de una hora, se plantearon las instrucciones del caso a resolver, los estudiantes formaron cinco grupos para su discusión y posteriormente se seleccionó al azar un estudiante como representante del grupo de tal forma que todos los estudiantes debían estar preparados para explicar la solución, a cada grupo se le planteo una pregunta detonante $¿$ el principal sospechoso miente o no miente?, justifique su respuesta. Como actividad de cierre se procedió a la presentación oral del caso y la retroalimentación final del mismo (conclusión general para toda la sección). 


\section{Determinemos la data de muerte mediante el uso de la entomofauna de interés médico legal}

Datos del Hallazgo (levantamiento del cadáver y colecta de indicios)

El 12 de diciembre del año 2015, a la 7.00 a.m., fue descubierto un cadáver en estado avanzado de putrefacción, con ropa masculina. En la región de la cabeza, pecho y abdomen se encontraron presencias de larvas de moscas (masa larval) y alrededor del cuerpo en menor cantidad. No se observan circunstancias irregulares.

El sitio del suceso es un espacio abierto, representado por un fragmento de bosque urbano (urbanización Yuma II) en el municipio San Diego, Estado Carabobo $\left(10^{\circ} 14^{\prime} 07^{\prime \prime}\right.$ Latitud Norte y $67^{\circ} 57 " 41^{* \prime}$ Latitud Oeste).

A las 8:00 a.m. se procedió a realizar la colecta de las larvas, siguiendo el protocolo establecido por Nuñez (2012). Se colectaron 30 larvas de la cabeza, pecho y abdomen, se sacrificaron, embalaron y trasladaron al laboratorio de entomología forense. Durante la colecta la temperatura promedio fue de $28^{\circ} \mathrm{C}\left(22-34^{\circ} \mathrm{C}\right) \mathrm{y}$ humedad relativa promedio $48 \%(25-71 \%)$.

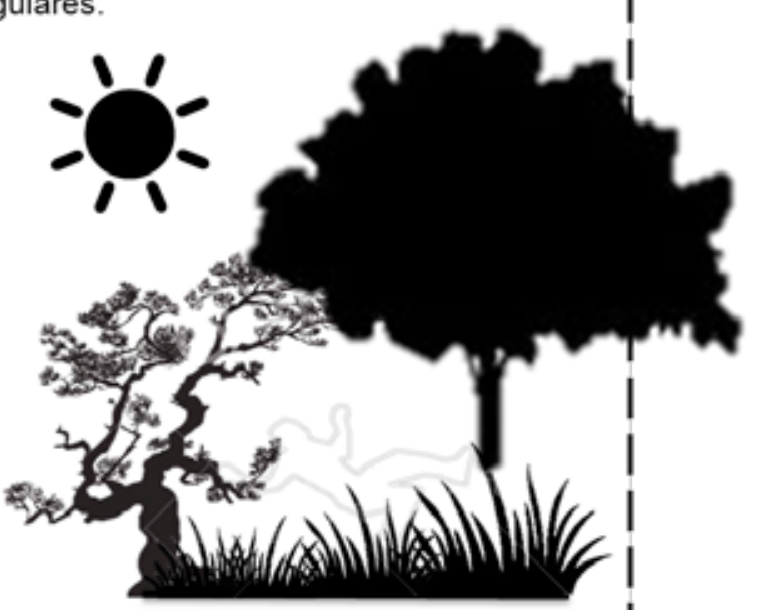

Datos de la investigación criminal (aportados por los investigadores)

«La familia lo vio por última vez la noche del 05 de diciembre del 2015 ".

«El principal sospechoso afirma haber visto a la victima con vida hace cuatro noches (miércoles 09 de diciembre del 2015, a las 08:00 pm)».

\section{Datos aportados por el patólogo y antropólogo forense}

Individuo de sexo masculino de aproximadamente 25 - 30 años de edad, mestizo. Cuerpo en estado avanzado de putrefacción (período colicuativo), el protocolo de necropsia revela un traumatismo en el cráneo realizado con un objeto contundente, con una data entre 6 y 7 dias.

Datos del laboratorio (aportados por el entomólogo forense)

- Las larvas fueron identificados como Chrysomya albiceps (Diptera: Calliphoridae), mediante el uso de las claves taxonómicas de Florez y Wolff (2009); Velásquez y otros (2010). Larvas de Instar III, longitud promedio $1,3 \mathrm{~cm}$.

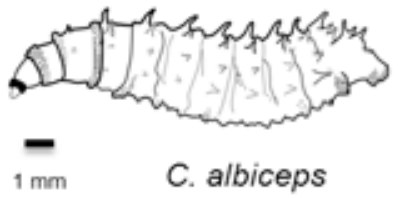

- Datos teóricos: En un laboratorio se determino que la especie C. albiceps requiere 124,7 horas a $30{ }^{\circ} \mathrm{C}$ para desarrollarse desde la etapa de huevo hasta la etapa de larva (Instar III).

Temp. min. de desarrollo $\left({ }^{\circ} \mathrm{C}\right): 10$

Datos de la temperatura del Instituto Nacional de Meteorologia e Hidrologia (INAMEH) para el Estado Carabobo. Disponible en: http://www.inameh.gob.ve/

\begin{tabular}{|c|c|c|c|}
\hline & \multicolumn{3}{c}{ Temperatura (aC) } \\
\hline Fecha & \multicolumn{1}{c|}{ Tempmax } & Temp min & Promedio \\
\hline $01 / 12 / 2015$ & 35 & 23 & 29 \\
\hline $02 / 12 / 2015$ & 32 & 23 & 27,5 \\
\hline $03 / 12 / 2015$ & 31 & 22 & 26,5 \\
\hline $04 / 12 / 2015$ & 33 & 22 & 27,5 \\
\hline $05 / 12 / 2015$ & 33 & 21 & 27 \\
\hline $06 / 12 / 2015$ & 33 & 23 & 28 \\
\hline $07 / 12 / 2015$ & 33 & 20 & 26,5 \\
\hline $08 / 12 / 2015$ & 33 & 22 & 27,5 \\
\hline $09 / 12 / 2015$ & 35 & 23 & 29 \\
\hline $10 / 12 / 2015$ & 34 & 24 & 29 \\
\hline $11 / 12 / 2015$ & 34 & 21 & 27,5 \\
\hline $12 / 12 / 2015$ & 34 & 22 & 28 \\
\hline $13 / 12 / 2015$ & 34 & 22 & 28 \\
\hline $14 / 12 / 2015$ & 33 & 23 & 28 \\
\hline
\end{tabular}

\section{Determine el IPM y redacte sus} conclusiones.

Fig. 4. Propuesta de caso problema para el aprendizaje de la entomología forense. 


\section{DISCUSIÓN DE RESULTADOS}

Tradicionalmente en Venezuela la data de muerte es determinada por un profesional en el campo de la medicina (médico patólogo forense) y al entrar en vigencia el manual del Ministerio Público abre un abanico de opciones para que los entomólogos forenses puedan prestar sus conocimientos al sistema de justicia. Recientemente en Venezuela, el Ministerio Público estableció el uso de la EF como un indicador de la data de muerte, al incluir en su Manual Único de Procedimientos en Materia de Cadena de Custodia de Evidencias Físicas (2012), que "se deberá verificar la data de muerte, mediante la evaluación de los fenómenos cadavéricos, tempranos y tardíos, así como desde el punto de vista de Entomología en caso de que existiera". Quiere decir que el Estado venezolano reconocer la importancia del uso de los insectos como evidencias y para ello se requiere profesionales capacitados en el análisis e interpretación de insectos asociados a cadáveres (Nuñez, 2012).

El método utilizado para resolver el caso planteado se denomina Horas grados acumulados (HGA), en el cual se requiere del conocimiento de las especies de moscas involucradas en los procesos de descomposición, su ciclo de vida, su relación con los distintos estados de la descomposición y con el hábitat en el cual son registradas (Gines y otros, 2015), por lo que es necesario contar con información básica que permita resolver el caso planteado. Al aplicar este método para determinar el IPM se utiliza la siguiente fórmula:

\section{HGA =Tiempo (horas) x (Temperatura-Temperatura base)}

Donde HGA: son las horas grados acumuladas necesarias para el desarrollo del insecto.

Tiempo (horas), es el tiempo de desarrollo que lleva el insecto. Es la incógnita del caso, porque se desea conocer cuando murió la persona.

Temperatura: es la temperatura a la cual se desarrolló el insecto, puede ser del sitio del suceso o la del laboratorio.
Temperatura base: es la temperatura más baja, en la cual no hay desarrollo del insecto (límite de temperatura para la supervivencia del insecto).

\section{Resolución del caso}

Para resolver el caso se hace necesario considerar la temperatura del sitio del suceso del día 12/12/15 y los días anteriores al hallazgo. Las horas de grado acumulados se calcularon con ayuda de la temperatura umbral para Chrysomya albiceps (Wiedemann, 1819) considerados como $10^{\circ} \mathrm{C}$ (Marchenko, 2001), con estos datos se calcularon las HGA (tabla II). El dato teórico de laboratorio es un patrón de referencia que permite comparar la realidad del caso con un control, dándole confiabilidad y validez a los resultados.

\begin{tabular}{|c|c|c|c|c|c|c|}
\hline \multirow[b]{2}{*}{ Fecha } & \multicolumn{3}{|c|}{ Temperatura $\left({ }^{\circ} \mathrm{C}\right)^{*}$} & \multirow{2}{*}{$\begin{array}{c}\text { Temp } \\
\text { Umbral } \\
\text { (C. albi- } \\
\text { ceps) }\end{array}$} & \multirow[b]{2}{*}{ HDA } & \multirow[b]{2}{*}{ HGA } \\
\hline & $\begin{array}{l}\text { Temp } \\
\text { máx. }\end{array}$ & $\begin{array}{l}\text { Temp } \\
\text { mín. }\end{array}$ & Prom & & & \\
\hline $1 / 12 / 2015$ & 35 & 23 & 29 & 10 & 19 & 456 \\
\hline $02 / 12 / 2015$ & 32 & 23 & 27,5 & 10 & 17,5 & 420 \\
\hline $03 / 12 / 2015$ & 31 & 22 & 26,5 & 10 & 16,5 & 396 \\
\hline 4/12/2015 & 33 & 22 & 27,5 & 10 & 17,5 & 420 \\
\hline $05 / 12 / 2015$ & 33 & 21 & 27 & 10 & 17 & 408 \\
\hline $6 / 12 / 2015$ & 33 & 23 & 28 & 10 & 18 & 432 \\
\hline 77/12/2015 & 33 & 20 & 26,5 & 10 & 16,5 & 396 \\
\hline $8 / 12 / 2015$ & 33 & 22 & 27,5 & 10 & 17,5 & 420 \\
\hline $99 / 12 / 2015$ & 35 & 23 & 29 & 10 & 19 & 456 \\
\hline $10 / 12 / 2015$ & 34 & 24 & 29 & 10 & 19 & 456 \\
\hline $11 / 12 / 2015$ & 34 & 21 & 27,5 & 10 & 17,5 & 420 \\
\hline $12 / 12 / 2015$ & 34 & 22 & 28 & 10 & 18 & 432 \\
\hline $13 / 12 / 2015$ & 34 & 22 & 28 & 10 & 18 & 432 \\
\hline $14 / 12 / 2015$ & 33 & 23 & 28 & 10 & 18 & 432 \\
\hline
\end{tabular}

*Datos obtenidos del Instituto Nacional de Meteorología e Hidrología (INAMEH) para el Estado Carabobo. Disponible en: http://www.inameh.gob.ve/

El tiempo de desarrollo desde la eclosión de los huevos hasta la emergencia de adultos de $C$. albiceps a temperatura constante de $30^{\circ} \mathrm{C}$ mediante el método 
HGA, en condiciones de laboratorio se presenta en la tabla III, tomado del trabajo de Al-Shareef y AlQurashi (2015) se calcularon las HGA.

Tabla III: Tiempo de desarrollo en horas para la especie $\mathrm{C}$. albiceps a temperatura constante de $30^{\circ} \mathrm{C}$ en condiciones de laboratorio.

\begin{tabular}{lll}
\hline \multicolumn{3}{c}{ C. albiceps, a $30^{\circ} \mathrm{C}$ y temperatura base $10^{\circ} \mathrm{C}$} \\
\hline Estadio & $\begin{array}{c}\text { Tiempo de } \\
\text { desarrollo (h) }\end{array}$ & HGA \\
Luevo & 9 & 180 \\
Larva I & 24 & 480 \\
Larva II & 24 & 480 \\
Pupa & 67,2 & 1344 \\
Adulto & 96 & 1920 \\
\hline Total & 200 & 4000 \\
\hline
\end{tabular}

Sobre la base de los datos anteriores, se calculó el IPM utilizando el método Horas Grado Acumulada como se muestra en la Tabla IV, para el diseño de la presentación didáctica de los cálculos el autor se apoyó del trabajo de Suri y otros, (2013).

Tabla IV: Determinación del IPM, mediante el método Horas Grados Acumulados.

\begin{tabular}{cc}
\hline Ítem & Resultado \\
\hline
\end{tabular}

El día 12 de diciembre del año 2015 a la 07.00 de la mañana (hora de la colecta

Colecta 8:00 am), se encuentra un cuerpo en estado avanzado de putrefacción. Presenta larvas cuya distribución es uniforme, colectándose 30 .

Fenómeno Cadáver en fenómeno colicuativo, data cadavérico aportada por el patólogo forense entre encontrado 6 y 7 días.

HGA necesarias para llegar

al Instar III, especie $\quad 180+480+480+1344=\mathbf{2 4 8 4}$ HGA Chrysomya albiceps a $30^{\circ} \mathrm{C}$

(Tabla III)
Total de HGA de los días 12 , $11,10,09,08 \mathrm{y}$ 07 de diciembre del 2015 (Tabla II)

Diferencia entre las HGA

División de las 192 HGA por el valor del HDA para el día 06/12/15 (Tabla II)

IPM

$$
\begin{gathered}
8 \times 18^{\circ} \mathrm{C}+24 \times 17,5^{\circ} \mathrm{C}+24 \times 19^{\circ} \mathrm{C} \\
+24 \times 19^{\circ} \mathrm{C}+24 \times 17,5^{\circ} \mathrm{C}+24 \times \\
16,5^{\circ} \mathrm{C}=2292 \text { HGA }
\end{gathered}
$$

$2484-2292=192$ HGA, le faltó a

la mosca para desarrollarse según las condiciones estándares de laboratorio.

$$
192 / 18=\mathbf{1 0 , 6} \text { horas }
$$

Entre la 1.00 y las 2.00 de la tarde del día 06 de diciembre del 2015.

Se concluye que la data de muerte del sujeto hallado el día 12 de diciembre del 2015 a las 7.00 am, murió entre la una y las dos de la tarde del día 06 de diciembre del año 2015, por lo cual el principal sospechoso miente. Este resultado se acerca a los resultados arrojados por la necropsia de ley, donde el patólogo forense concluye una data de muerte entre 6 y 7 días.

\section{Descripción de la aplicación del método de casos propuesto para el aprendizaje de la EF y resultados:}

Durante la primera fase se entregó el caso a los cinco grupos conformados, los mismos analizaron la información plasmada en el caso e identificaron los elementos de interés criminalísticos aportados. La pregunta principal fue ¿Cuándo murió la persona?; es decir, determinar la data de muerte mediante el uso de insectos aplicando el método HGA. Los equipos analizaron cada detalle apoyándose en los aspectos teóricos vistos durante la clase y los conocimientos previos adquiridos a lo largo del diplomado. Durante la segunda fase los estudiantes seleccionados de cada equipo presentaron de forma oral sus soluciones al caso planteado, lo cual favorece el desarrollo de las facilidades de comunicación, punto importante por ser el juicio oral y público; durante esta presentación oral 
sospechoso miente o no miente cuando aseguro haber visto al hoy occiso con vida la noche del día 09 de diciembre del 2015?, cada grupo defendió su punto de vista y como actividad de cierre se realizó una conclusión general de toda la sección posterior al análisis detallado del caso, resumiendo los resultados de los cinco grupos en la tabla V.

Tabla V: Respuesta y conclusiones según cada grupo.

\begin{tabular}{|c|c|}
\hline Grupo & Respuesta \\
\hline 01 & $\begin{array}{l}\text { "La data de muerte estimada fue el } 06 / 12 / 15 \text { a } \\
\text { la } 01: 00 \mathrm{pm} \text {, por lo que se comprueba que el } \\
\text { principal sospechoso miente al decir que vio a la } \\
\text { víctima con vida hace cuatro noches" }\end{array}$ \\
\hline 02 & $\begin{array}{l}\text { "El sospechoso miente, ya que según las } \\
\text { investigaciones la persona falleció el } 06 / 12 / 15 \text { a } \\
\text { las 12:40 pm aproximadamente" }\end{array}$ \\
\hline 03 & $\begin{array}{l}\text { "La data de muerte del sujeto se estima que } \\
\text { ocurrió entre la } 1: 00 \text { a } 2: 00 \mathrm{pm} \text { del } 06 / 12 / 2015 " .\end{array}$ \\
\hline 04 & $\begin{array}{l}\text { "El sospechoso miente ya que según el estudio } \\
\text { entomológico de las larvas encontradas, la } \\
\text { mosca se posó en el cadáver aproximadamente } \\
\text { entre la } 1 \text { a } 2 \text { de la tarde del día } 06 / 12 / 15 \text {, por lo } \\
\text { cual no lo pudo haber visto con vida hace cuatro } \\
\text { noches" }\end{array}$ \\
\hline 05 & $\begin{array}{l}\text { "La data de muerte aproximada es que la persona } \\
\text { murió el } 06 / 12 / 2015 \text { a la 01:00 pm, por lo tanto } \\
\text { el sospechoso no pudo haber visto a la víctima } \\
\text { con vida hace cuatro noches; en conclusión está } \\
\text { mintiendo. }\end{array}$ \\
\hline $01-($ & $\begin{array}{l}\text { Los cinco grupos concluyeron posterior al } \\
\text { análisis crítico de cada resultado que "la data } \\
\text { de muerte mediante el método Horas Grados } \\
\text { Acumulados (HGA) fue el día } 06 / 12 / 15 \text {, la } \\
\text { primera mosca que llego al cuerpo fue C. } \\
\text { albiceps entre la una y las dos de la tarde de ese } \\
\text { día; por lo cual el sospechoso miente al afirmar } \\
\text { que vio al hoy occiso la noche del día } 09 \text { de } \\
\text { diciembre del } 2015 \text { ". } \\
\text { "Con respecto a la data arrojada por el patólogo } \\
\text { forense se ajusta a nuestro resultado; sin } \\
\text { embargo, nosotros no podemos asegurar que el } \\
\text { sujeto murió antes de la una de la tarde del } 12 \text { de } \\
\text { diciembre, porque nuestro resultado se basa en } \\
\text { el estudio de una mosca". }\end{array}$ \\
\hline
\end{tabular}

Fuente: elaboración, basado en las conclusiones de los cinco grupos.
Se evidencio que los estudiantes lograron resumir un caso con mucha información en un resultado puntual que permitirá mejorar el sistema de justicia al ser precisos y objetivos con sus conclusiones durante el análisis de una evidencia. Ciertamente las cinco conclusiones se aproximan a la conclusión planteada en la tabla IV, se puede observar que este tipo de estrategia de aprendizaje, requiere una explicación detallada sobre los objetivos que se buscan lograr; así como todos los fundamentos teóricos necesarios para generar una respuesta, la idea es no crear frustración en el estudiante por la falta de información sobre todo al momento de aplicar las matemáticas durante el cálculo para determinar la data de muerte porque pierden la motivación, pero también es necesario que los casos los hagan pensar; la idea es apoyar los procesos de compresión que realizan los estudiantes, siendo una estrategia que combina la investigación con el trabajo de campo y laboratorio, que es común para todo criminalista o investigador forense. En la figura 5 se resume los procesos de aprendizaje observados en los cinco grupos mediante el método de caso.

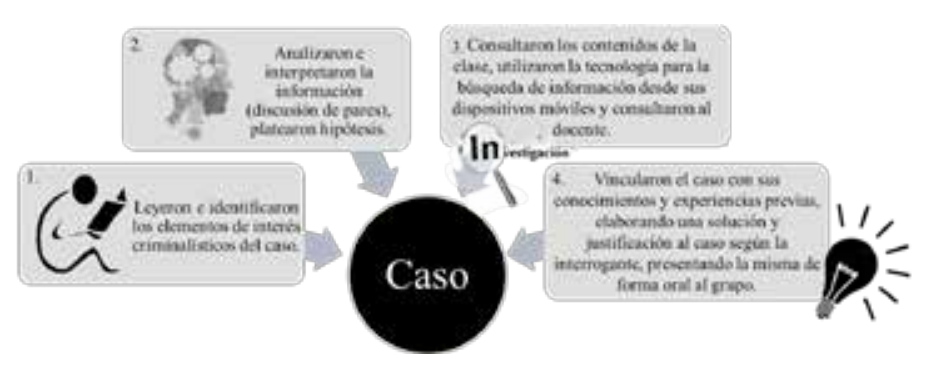

Figura 5: Resumen del proceso de aprendizaje mediante el método de caso, observado en los participantes. Elaboracion propia.

Se evidencio que analizar los casos en grupos pequeños fomenta el aprendizaje colaborativo, el docente es un mediador pedagógico durante el proceso, por lo cual es una estrategia que permite valorar si el estudiante realmente está aprendiendo al momento de presentar su caso (aprendizaje significativo), como se expresa, como defiende su punta de vista, como fundamenta cada caso con los conocimientos previos y la información que comparte con el grupo, desarrollando 
sus competencias. Para los docentes este tipo de estrategia fomenta la investigación y el análisis para lograr ofrecer cuales son las posibles soluciones que sus estudiantes podrían plantear, permitiendo construir y reconstruir el conocimiento, flexibilizando su punto de vista.

En general los estudiantes aprenden tanto los contenidos teóricos que fundamentan sus conclusiones, así como de la experiencia de ponerlos en práctica durante el análisis del caso. Para González (2013), en el mundo profesional no será suficiente el dominio de los conceptos teóricos para operar eficazmente, mucho menos en un campo como el de la criminalística y ciencias forenses donde la diversidad de casos es muy evidente; por lo cual será necesario contar con las habilidades para saber aplicarlos y la actitud que se toma al ponerlos en práctica; es decir, que también deben desarrollar un juicio profesional ético y responsable aunado a una competencia práctica.

\section{CONCLUSIONES}

Como conclusión se observa que el uso de métodos de casos como estrategia de aprendizaje aplicado en la $\mathrm{EF}$, permite trabajar en pequeños grupos para analizar, comparar y contrastar posibles soluciones con las soluciones de otros; esto es importante porque la data de muerte aportada por el entomólogo forense puede diferir con la data de muerte aportada por el patólogo forense o el antropólogo forense y esto se debe a que el entomólogo forense utiliza como evidencia para su análisis los insectos (larvas) colectados directamente del cuerpo, estimando el tiempo de vida del insecto lo cual se traduce en el tiempo mínimo de muerte del sujeto. Un tiempo mayor a este resultado el entomólogo no puede aportar dicha información, pero se puede justificar de la siguiente manera (Magaña, 2001; Nuñez, 2012):

- Que el cuerpo haya sido quemado, enterrado, sumergido completamente.
- Que el cuerpo haya sido conservado mediante la refrigeración o embalsamamiento.

- Que el cuerpo haya sido envuelto en bolsas plásticas, sabanas, también influye el tipo de vestimenta.

- Que el cuerpo haya sido trasladado del lugar.

- Que el lugar del fallecimiento sea lo suficientemente oscuro e inaccesible para los insectos, ejemplo dentro de una casa, un carro.

- Que los restos de los dípteros hayan desaparecidos por la acción de los necrófilos.

- Que el cadáver haya sido impregnado con productos repugnatorios (sustancias tóxicas, por ejemplo, insecticidas), impidiendo la colonización del cuerpo.

\section{- Entre otras causas.}

Desde el punto de vista educativo este tipo de estrategias desarrolla actitudes positivas hacia la tolerancia, dadas las diversas soluciones que pueden aportar cada equipo; Sin embargo, recordemos que desde el punto de vista criminalístico y de las ciencias forenses debe existir una solución que se ajuste a la verdad procesal basados en los diversos métodos de la criminalística, de allí surge la importancia de la aplicación de las ciencias y disciplinas auxiliares de la criminalística, apoyadas en el debate de ideas durante la formación académica de los estudiantes.

Los estudiantes durante la aplicación del método de caso desarrollaron las competencias para analizar los problemas en el campo de la EF, determinando los métodos de análisis según sus conocimientos y experiencias, esto les permitió analizar, investigar, proponer alternativas y tomar decisiones, fomentando la responsabilidad, el compromiso grupal, la reflexión y el pensamiento crítico, cualidades que deben poseer los investigadores forenses, la idea del método de caso es que los estudiantes ponga a prueba sus argumentos y profundicen el análisis del problema planteado.

Dentro de las habilidades que desarrollaron los estudiantes se destacan: la analítica al identificar el 
problema, la toma de decisiones al proponer una solución, habilidades para la comunicación oral $\mathrm{y}$ escrita, defendiendo y argumentando sus conclusiones, reconociendo sus errores y debilidades; por último, el manejo del tiempo y el trabajo en equipo les permitió aportar sus conclusiones en plazos que dentro del proceso penal son vitales. Para los estudiantes resulto ser una estrategia innovadora que los motivo durante el dictado del módulo.

\section{AGRADECIMIENTOS}

A los participantes del Diplomado en Ciencias Forenses y Criminalística de la Universidad José Antonio Páez, Cohorte IX-Diciembre 2015, por la motivación durante todo el diplomado y el permiso para la publicación de esta experiencia.

\section{REFERENCIAS BIBLIOGRÁFICAS}

Al-Shareef, L. y Al-Qurashi, S. (2015). Study of some biological aspects of the blowfly Chrysomya albiceps (Wiedemann, 1819) (Diptera: Calliphoridae) in Jeddah, Saudi Arabia. Egyptian Journal of Forensic Sciences. [Documento en línea]. Disponible: http://dx.doi.org/10.1016/j. ejfs.2015.06.003. [Consulta: 2015, diciembre 01]. Amendt, J., Lee Goff, M., Campobasso C., y Grassberger, M. (2010). Current Concepts in forensic entomology. Springer Dordrechet Heidelberg London New York. 363 p.

Bersanelli, M. y Gargantini, M. (2006). Sólo el asombro conoce. La aventura de la investigación científica. $1^{a}$ Edición. Ediciones Encuentro. Madrid, 358p.

Bonfigli, E., Trujillo, E., Cantín M. y Fonseca, G. (2010). Procedimientos y aprendizaje significativo en la investigación criminal. Presentación de dos experiencias de capacitación interdisciplinaria. The Forensic Oral Pathology Journal, 1(2): 14-19.

Camacho, G. (2005). Manual de recolección, preservación y embalaje de muestras entomológicas en el lugar de los hechos en Bogotá D.C. Tesis de Postgrado. Facultad de Investigación Criminal.
Escuela Nacional de Policía General Santander, 73 p.

Florez, E. y Wolff, M. (2009). Descripción y clave de los estadios inmaduros de las principales especies de Calliphoridae (Diptera) de importancia forense en Colombia. Neotropical Entomology, 38(3): 418-429.

García, F. (2005). La serie C.S.I. como metáfora de algunas facetas del trabajo científico. Revista Eureka sobre Enseñanza y Divulgación de las Ciencias, 2(3): 374-387.

García, J., Sánchez, C., Jiménez, M. y Gutiérrez, M. (2012). Estilos de Aprendizaje y Estrategias de Aprendizaje: un estudio en discentes de postgrado. Revista Estilos de Aprendizaje, 10(10): 1-17.

Gennard, D. (2007). Forensic entomology. An introduction. John Wiley \& Sons Ltd, The Atrium, Southern Gate, Chichester, England, 170 p.

Gines, E., Alcántara, M., Calderón, C., Infante, C. y Villacorta, M. (2015). Entomofauna de interés forenses asociada a restos cadavéricos de cerdos (Sus scrofa L.), expuestos a condiciones de campo en Lambayeque-Perú. Revista Peruana de Entomología, 50(1): 1-11.

González, J. (2013). El método del caso. Multidisciplinary Business Review, 6(2): 1-5.

González, E. (2015). Estudio de casos como estrategia didáctica en la formación del estudiantado en Bibliotecología. Revista Electrónica E-Ciencias de la Información, 5(2): 1-15.

Greenberg, B. (1990). Behavior of postfeeding larva of some Calliphoridae and a muscid (Diptera). Annals of the Entomological Society of America, 83: $1210-1214$.

Magaña, C. (2001). La entomología forense y su aplicación a la medicina legal. Data de la muerte. Aracnet 7 -Bol. S.E.A, 28: 49-57.

Marchenko, M. (2001). Medicolegal relevance of cadáver entomofauna for the determination of the time of death. Forensic Science International, 120: 89-109.

Mavárez M., Espina A., Barrios F. y Fereira J. (2005). La Entomologia forense y el neotrópico. Cuaderno 
de Medicina Forense, 11: 22-33.

Ministerio Público. (2012). Manual Único de Procedimientos en Materia de Cadena de Custodia de Evidencias Físicas. Caracas, 309p.

Niño, L. (2012). Estudio de caso: una estrategia para la enseñanza de la educación ambiental. Praxis \& Saber, 3(5): 53-78.

Nuñez, J. (2012). Entomología forense en Venezuela. $1^{a}$ Edición. Editorial Livrosca. Caracas, 273 p.

Nuñez, J. (2015). Didáctica del módulo de microanálisis del Diplomado en Ciencias Forenses y Criminalística de la Universidad José Antonio Páez. Otenido de: http://w3.ujap.edu.ve/pasion/ index.php/89-edicion8/173-didactica-del-modulode-microanalisis-del-diplomado-en-cienciasforenses-y-criminalistica.

Ramírez, J. (2012). Diccionario entomológico venezolano. Ediciones IVIC. Caracas, 350p.

Rivas, E. (2006). Estadística General. 11 ${ }^{\mathrm{a}}$ Edición. Universidad Central de Venezuela, Caracas, 310p.

Sanabria, C. (2008). Antropología forense y la investigación médico legal de las muerte. $2^{\mathrm{a}}$ Edición. Asociación Colombiana de Antropología Forense. Colombia, 432 p.

Sebastiany, S., Pizzato, M., Diehl, I. y Miskinis, T. (2013a). Aprendiendo a investigar por medio de la
Ciencia forense e investigación criminal. Revista Eureka sobre Enseñanza y Divulgación de las Ciencias, 10(3): 480-490.

Sebastiany, S., Pizzato, M., Del Pino, J. y Miskinis, T. (2013b). A utilização da Ciência Forense e da Investigação Criminal como estratégia didática na compreensão de conceitos científcos. Educación química, 24(1): 49-56.

Sukontason, K., Sukontason, K., Piangjai, S., Boonchu, N., Kurahashi, H., Hope, M. y Olson, J. (2004). Identification of forensically eggs using a potassium permanganate staining technique. Micron, 35: 391-395.

Suri, B., Sharma, H. y Bharti, M. (2013). Estimation of post mortem interval by rearing Chrysomya rufifacies (Macquart, 1842) (Diptera: Calliphoridae): A case study from central India. Journal of Forensic Medicine and Toxicology, 14(2): 1-12.

Tamayo, M. (2003). El Proceso de la Investigación Científica. $4^{\mathrm{a}}$ Edición. Limusa, Noriegas Editores. México, 425p.

Velásquez, Y., Magaña, C., Martinez, A. y Rojo, S. (2010). Diptera of forensic importance in the Iberian Peninsula: larval identification key. Medical and Veterinary Entomology. 24:11-16. 